\title{
La malnutrición infantil en El Oro: una paridad asimétrica
}

\section{Child malnutrition in El Oro: an asymmetric parity}

\author{
Jairo Rivera \\ Universidad Andina Simón Bolívar, \\ Quito-Ecudor \\ hjairo.rivera@uasb.edu.ec \\ https://orcid.org/0000-0003-3528-5969 \\ Stephany Olarte \\ Universidad Técnica Particular de Loja, \\ Quito-Ecuador \\ scolarte@utpl.edu.ec \\ https://orcid.org/0000-0002-1258-6913
}

Nadya Rivera Universidad Tecnológica Equinoccial, Quito-Ecuador nrivera@ute.edu.ec https://orcid.org/0000-0003-1520-8499

\section{Revista Cumbres Vol.7 №2}

Versión electrónica ISSN 1390-3365 http://investigacion.utmachala.edu.ec/revistas/index.php/Cumbres http://doi.org/10.48190/cumbres.v7n2a1 


\title{
RESUMEN
}

La malnutrición infantil en Ecuador refleja un contexto complejo, donde los indicadores de desnutrición y sobrepeso son mayores al promedio de América Latina. El objetivo de este documento es examinar la evolución de la malnutrición infantil en El Oro, sus particularidades, progresos y desafíos. La metodología utilizada es cuantitativa en base a los datos de las encuestas de condiciones de vida 1999-2006-2014, y se realizan estimaciones mediante modelos probabilísticos como análisis de regresión. Los principales resultados indican que, aunque los niveles de desnutrición y sobrepeso son bastante similares en $17 \%$, presentan distintos determinantes sociales y personales, los cuales cambian en el tiempo. En el caso de la desnutrición infantil, los determinantes se relacionan con la pobreza, el sexo, el orden de nacimiento y la edad de la madre; por su parte, los determinantes del sobrepeso se asocian con la pobreza y el orden de nacimiento.

Palabras clave: desnutrición, sobrepeso, análisis de regresión.

\begin{abstract}
Child malnutrition in Ecuador reflects a complex context, where indicators of stunting and overweight are higher than the Latin American average. The objective of this document is to examine the evolution of child malnutrition in El Oro, its peculiarities, progress and challenges. The methodology used is quantitative based in the data from the Living Conditions Surveys 19992006-2014, and makes estimates using probabilistic models as regression analysis. The main results indicate that although the levels of stunting and overweight are quite similar in 17\%, they present different social and personal determinants, which change over time. In the case of child malnutrition, the determinants are related to poverty, sex, birth order and the mother's age; on the other hand, the determinants of overweight are associated with poverty and birth order birth.
\end{abstract}

Keywords: malnutrition, overweight, regression analysis. 


\section{INTRODUCCIÓN}

La malnutrición infantil, entendida como la desnutrición y el sobrepeso, afecta el desarrollo de los menores de cinco años, alrededor del mundo, limitando sus capacidades, teniendo repercusiones en la sociedad y en las generaciones futuras (Sundaram, 2015). En ese sentido, los costos de la malnutrición infantil "son extremadamente elevados" (Martínez y Fernández 2009).

Las causas de la malnutrición son multidimensionales y se relacionan con factores ambientales, sociales y personales; es decir, obedecen a temas del entorno socioeconómico, pobreza, acceso a servicios públicos, alimentación, prácticas de lactancia, alimentación complementaria, entre otros (OPS, 2008); asimismo, se asocian con estados de salud intertemporales, donde la existencia o ausencia de problemas nutricionales en la madre embarazada pueden afectar el estado nutricional del bebé (Berlinski y Schady, 2015). Estos factores dependen de cada contexto, no son homogéneos entre países, regiones, ni ciudades, para lo cual es fundamental realizar un estudio sobre los determinantes de un contexto específico que sea de interés y que ello sirva de base para el desarrollo de políticas públicas.

En el Ecuador, la prevalencia de desnutrición es extremadamente alta en relación con los países de América Latina, duplicando el promedio de la región (Rivera, 2019). En su tendencia, la malnutrición infantil ha tenido una limitada reducción de la desnutrición y un crecimiento del sobrepeso infantil (Freire, 2014). En esa línea, los progresos de malnutrición infantil en el país denotan un panorama complejo, que se alejan del cumplimiento de los Objetivos de Desarrollo Sostenible elaborados por las Naciones Unidas, con lo que "no será suficiente para alcanzar la meta que plantea reducir esta condición a la mitad para el 2021" (Chavez y col., 2020).

Dentro del Ecuador, las prevalencias de desnutrición y sobrepeso son diversas (Freire, 2013). A su vez, dichos niveles de malnutrición infantil se asocian con determinantes diversos, los cuales responden a contextos locales (Rivera y Olarte, 2020; Rivera, Olarte y Rivera, 2020). Por ello, el objetivo de esta investigación es examinar el estado de la malnutrición infantil en la provincia de El Oro y encontrar los determinantes que afectan a la desnutrición infantil y al sobrepeso infantil. Para ello se utiliza una metodología cuantitativa, fundamentada en las encuestas de condiciones de vida.

\section{MATERIALES Y MÉTODOS}

La investigación se fundamenta en la Encuesta de Condiciones de Vida (ECV), producida por el Instituto Nacional de Estadística y Censos del Ecuador. Dicha encuesta tiene libre acceso en el portal web del INEC, con representatividad nacional, regional, y provincial. Desde finales de la década de los 
noventa incluye información nutricional, con lo cual se utiliza las encuestas de 1999 (5816 viviendas), 2006 (13581 viviendas), y 2014 (29052 viviendas). En la estimación se utiliza un modelo probabilístico PROBIT, a través del software STATA, con el fin de estimar los determinantes que afectan la probabilidad de tener desnutrición o sobrepeso, mediante las curvas de crecimiento de la OMS (OMS, 2006). En este modelo se obtienen los resultados marginales de la estimación. Con ello, se estima la siguiente ecuación:

$$
\mathrm{Y}_{\mathrm{i}}=\rho \mathrm{XAmbientales}_{\mathrm{i}}+\gamma \text { XSociales }_{\mathrm{i}}+\varphi \text { XPersonales }_{\mathrm{i}}+\varepsilon_{\mathrm{i}}
$$

donde Y es una variable binaria que toma el valor de 1 si la persona tiene desnutrición crónica (sobrepeso) y 0 si la persona no tiene. Dentro de las variables explicativas se encuentran: XAmbientales es un vector de variables ambientales que incluye: área; XSociales es un vector de variables sociales que incluye: pobreza por consumo, agua por red pública, servicio sanitario y alcantarillado, recolección de basura, tipo de techo, tipo de paredes, tipo de piso, recibe vitamina A, y recibe hierro; XPersonales es un vector de variables personales que incluye: sexo, edad, bajo peso, presencia de diarrea, presencia de enfermedades respiratorias, carné de salud, matrícula en guardería, prácticas de lactancia correctas, cuidado de la madre, trabajo madre, deporte madre, orden de nacimiento, edad de la madre y educación de la madre; con sus respectivos parámetros, $\rho, \gamma, \varphi$.

\section{RESULTADOS Y DISCUSIÓN}

Las estadísticas descriptivas de la provincia de El Oro, en base a las ECV, se presentan en la Tabla 1. En términos generales se observa una mejora dentro de las condiciones de vida de la población. El Oro es una provincia con "alto potencial productivo" (Prefectura de El Oro, 2015), mayoritariamente urbana, con cerca de tres cuartos de la población en esa área, donde los indicadores de bienestar dentro de la provincia han mejorado notablemente (INEC, 2010). En ese sentido, existen algunos indicadores como educación donde la provincia de El Oro presenta mejores índices que el promedio nacional (Senplades, 2014). La pobreza por consumo se ha reducido gradualmente y es inferior al promedio nacional. Las coberturas de servicios se han incrementado en agua por red pública, alcantarillado y recolección de basura. La prevalencia de enfermedades ha decrecido en enfermedades como diarrea y enfermedades respiratorias. La obtención del carné de salud se ha incrementado, lo mismo que sucede con la matrícula en la guardería, los años de educación de la madre y la proporción de madres que tiene un trabajo.

\section{Cumbres}


Tabla 1.

Estadísticas descriptivas

\begin{tabular}{|c|c|c|c|c|}
\hline & & & AÑOS & \\
\hline Variables & & 1999 & 2006 & 2014 \\
\hline \multirow[t]{2}{*}{ Área rural } & Sí & 36,5 & 22,0 & 23,7 \\
\hline & No & 63,5 & 78,0 & 76,3 \\
\hline \multirow[t]{2}{*}{ Pobreza consumo } & Sí & 77,8 & 35,9 & 30,8 \\
\hline & No & 22,2 & 64,1 & 69,2 \\
\hline \multirow[t]{2}{*}{ Agua red pública } & Sí & 52,3 & 81,5 & 84,4 \\
\hline & No & 47,7 & 18,5 & 15,6 \\
\hline \multirow[t]{2}{*}{ Servicio sanitario y alc. } & Sí & 43,8 & 61,0 & 67,3 \\
\hline & No & 56,2 & 39,0 & 32,7 \\
\hline \multirow[t]{2}{*}{ Recolección basura mun. } & Sí & 50,1 & 84,1 & 94,4 \\
\hline & No & 49,9 & 15,9 & 5,6 \\
\hline \multirow[t]{2}{*}{ Techo adecuado } & Sí & 27,7 & 27,7 & 30,6 \\
\hline & No & 72,3 & 72,3 & 69,4 \\
\hline \multirow[t]{2}{*}{ Paredes adecuadas } & Sí & 75,6 & 81,3 & 87,9 \\
\hline & No & 24,4 & 18,7 & 12,1 \\
\hline \multirow[t]{2}{*}{ Piso adecuado } & Sí & 3,8 & 15,6 & 28,0 \\
\hline & No & 96,2 & 84,4 & 72,0 \\
\hline \multirow[t]{2}{*}{ Vitamina A } & Sí & 18,5 & 22,1 & 51,9 \\
\hline & No & 81,5 & 78,0 & 48,1 \\
\hline \multirow[t]{2}{*}{ Hierro } & Sí & 8,5 & 14,0 & 48,4 \\
\hline & No & 91,5 & 86,0 & 51,6 \\
\hline \multirow[t]{2}{*}{ Mujer } & Sí & 53,1 & 51,8 & 46,8 \\
\hline & No & 46,9 & 48,2 & 53,2 \\
\hline \multirow[t]{2}{*}{ Bajo peso } & Sí & 0,0 & 1,7 & 5,7 \\
\hline & No & 100,0 & 98,3 & 94,3 \\
\hline \multirow[t]{2}{*}{ Diarrea } & Sí & 29,8 & 25,1 & 20,1 \\
\hline & No & 70,2 & 74,9 & 79,9 \\
\hline \multirow[t]{2}{*}{ Enf. Respiratorias } & Sí & 65,6 & 56,1 & 47,4 \\
\hline & No & 34,4 & 43,9 & 52,6 \\
\hline \multirow[t]{2}{*}{ Carné de salud } & Sí & 62,2 & 78,2 & 86,1 \\
\hline & No & 37,8 & 21,8 & 13,9 \\
\hline \multirow[t]{2}{*}{ Matriculado guardería } & Sí & 8,6 & 34,8 & 49,5 \\
\hline & No & 91,4 & 65,2 & 50,5 \\
\hline \multirow[t]{2}{*}{ Lactancia correcta } & Sí & 5,4 & 9,3 & 10,4 \\
\hline & No & 94,7 & 90,7 & 89,6 \\
\hline \multirow[t]{2}{*}{ Cuidado madre } & Sí & 83,3 & 76,0 & 84,2 \\
\hline & No & 16,7 & 24,0 & 15,8 \\
\hline \multirow[t]{2}{*}{ Trabaja madre } & Sí & 24,7 & 48,9 & 44,7 \\
\hline & No & 75,3 & 51,1 & 55,3 \\
\hline
\end{tabular}




\begin{tabular}{|lllll|}
\hline Deporte madre & Sí & 4,8 & 8,7 & 20,5 \\
\hline & No & 95,2 & 91,3 & 79,5 \\
\hline Orden de nacimiento & Media & 3,5 & 2,5 & 2,6 \\
\hline Edad meses del menor & Media & 35,4 & 29,5 & 29,6 \\
\hline Educación madre & Media & 9,6 & 10,8 & 11,4 \\
\hline Edad madre & Media & 31,3 & 27,7 & 29,0 \\
\hline
\end{tabular}

La figura 1 muestra la evolución de la malnutrición infantil en la provincia de El Oro durante las últimas dos décadas. En el tiempo, la desnutrición crónica y el sobrepeso infantil no presentan una tendencia clara, con varios movimientos, aunque al comparar todo el periodo se muestra que la desnutrición disminuye y el sobrepeso aumenta, ya que en 1999 alrededor del 23 \% de menores de cinco años tenía desnutrición y en 2018 se ubica en 17\%, mientras que el sobrepeso se ubicaba en 3\% en 1999, y asciende a $17 \%$ en el año 2018. Resulta interesante resaltar que la aparente paridad entre ambos indicadores es única en el país.

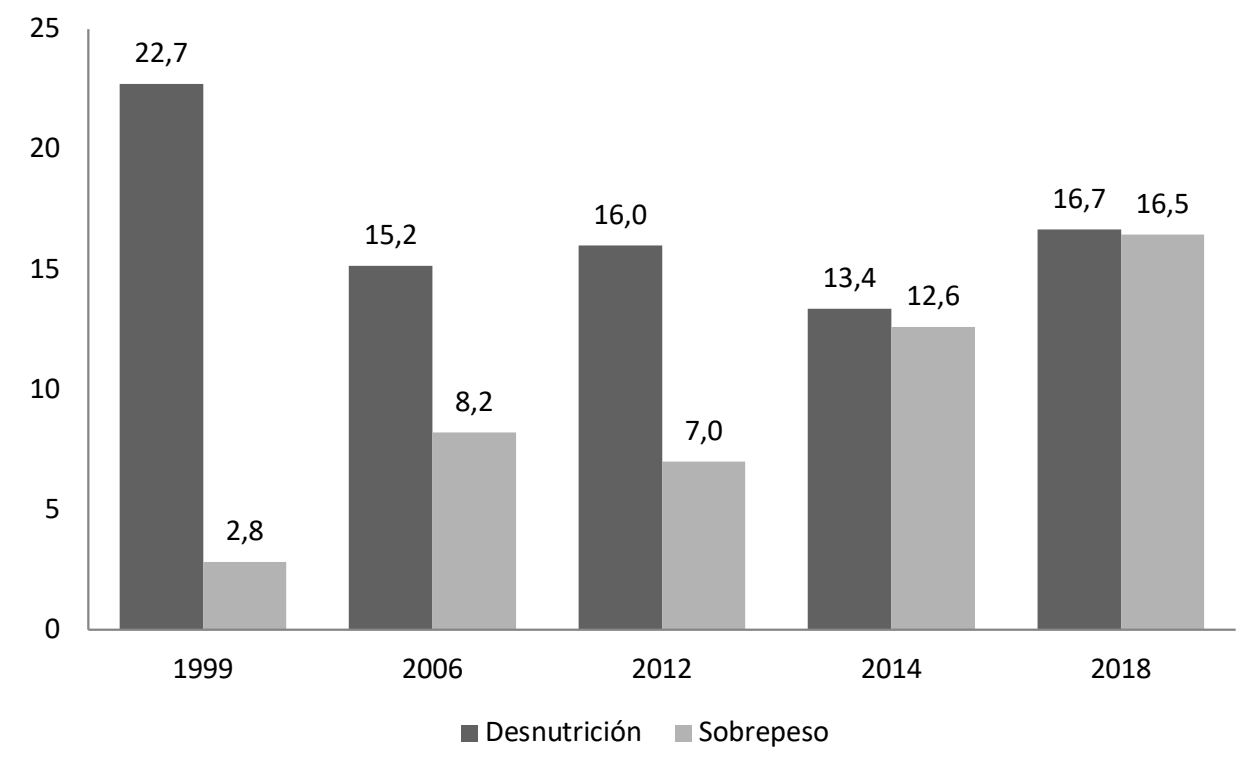

Figura 1. La malnutrición infantil en El Oro

Por una parte, en la Tabla 2, para el año 2014, se halla que la probabilidad de tener desnutrición en El Oro se asocia con la pobreza, el sexo, el orden de nacimiento y la edad de la madre. En ese sentido, la desnutrición tiene relación positiva con la pobreza y el orden de nacimiento; la explicación es simple y es que a mayor pobreza se torna compleja la satisfacción de necesidades nutricionales, y a la vez, ante un mayor número de niños, es difícil atender satisfactoriamente sus requerimientos nutritivos. Al mismo tiempo, la desnutrición presenta una relación inversa con la edad de la madre y el sexo fe- 
menino; la ilustración de estas asociaciones se relaciona con la cada vez más difícil práctica de cuidado desde la madre, donde las madres jóvenes tienen complejidades en el cuidado de la nutrición infantil, siendo el problema aún más grave en los hombres.

Tabla 2.

Determinantes de la malnutrición infantil en El Oro

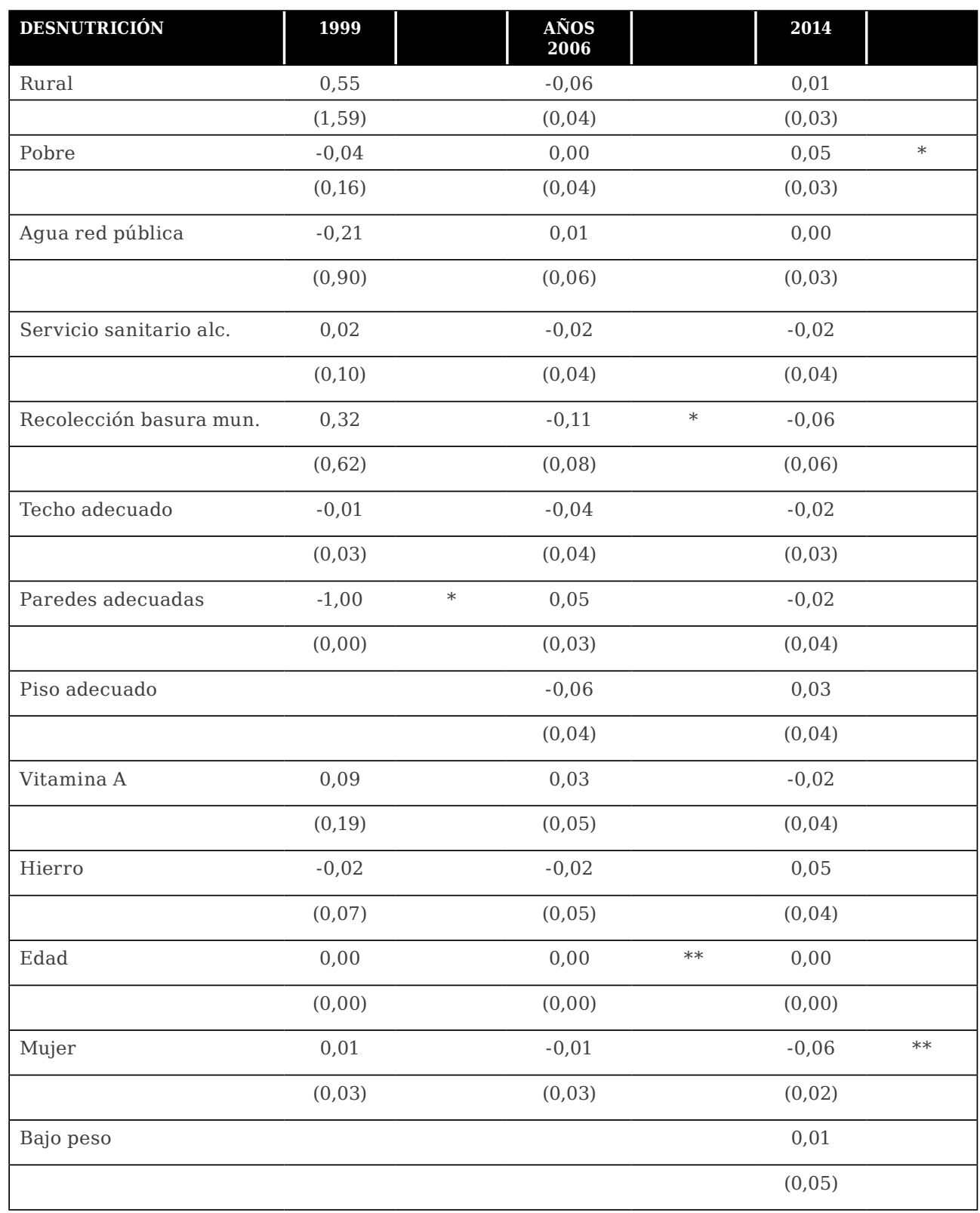




\begin{tabular}{|c|c|c|c|c|c|}
\hline Orden nacimiento & 0,02 & 0,02 & & 0,04 & $* * *$ \\
\hline & $(0,07)$ & $(0,01)$ & & $(0,01)$ & \\
\hline \multirow[t]{2}{*}{ Diarrea } & $-1,00$ & $-0,04$ & & $-0,03$ & \\
\hline & $(0,01)$ & $(0,03)$ & & $(0,03)$ & \\
\hline \multirow[t]{2}{*}{ Enf. Respiratorias } & 0,03 & 0,01 & & $-0,01$ & \\
\hline & $(0,06)$ & $(0,03)$ & & $(0,03)$ & \\
\hline \multirow[t]{2}{*}{ Carné salud } & 0,04 & $-0,09$ & $*$ & 0,01 & \\
\hline & $(0,11)$ & $(0,05)$ & & $(0,04)$ & \\
\hline \multirow[t]{2}{*}{ Matriculado guardería } & & $-0,02$ & & 0,01 & \\
\hline & & $(0,04)$ & & $(0,03)$ & \\
\hline \multirow[t]{2}{*}{ Edad madre } & 0,00 & $-0,01$ & $* * *$ & $-0,01$ & $* *$ \\
\hline & $(0,00)$ & $(0,00)$ & & $(0,00)$ & \\
\hline \multirow[t]{2}{*}{ Educación madre } & $-0,01$ & $-0,01$ & & 0,00 & \\
\hline & $(0,03)$ & $(0,01)$ & & $(0,00)$ & \\
\hline \multirow[t]{2}{*}{ Lactancia correcta } & & $-0,03$ & & 0,04 & \\
\hline & & $(0,05)$ & & $(0,05)$ & \\
\hline \multirow[t]{2}{*}{ Cuidado madre } & 0,00 & 0,01 & & 0,04 & \\
\hline & $(0,02)$ & $(0,04)$ & & $(0,03)$ & \\
\hline \multirow[t]{2}{*}{ Trabaja madre } & 0,01 & $-0,03$ & & 0,01 & \\
\hline & $(0,17)$ & $(0,04)$ & & $(0,03)$ & \\
\hline \multirow[t]{2}{*}{ Deporte madre } & & $-0,07$ & & 0,00 & \\
\hline & & $(0,04)$ & & $(0,03)$ & \\
\hline $\mathbf{N}$ & 56 & 316 & & 661 & \\
\hline Pseudo R2 & 0,67 & 0,19 & & 0,08 & \\
\hline Prob. Neta Predicha & 0,89 & 0,86 & & $\mathbf{0 , 8 7}$ & \\
\hline
\end{tabular}

En referencia al sobrepeso infantil en El Oro, cuyos resultados se presentan en la tabla 3, se encuentra que la probabilidad de padecerla se relaciona con factores asociados con la pobreza y el orden de nacimiento. En esa línea, la probabilidad de sobrepeso infantil se incrementa si el hogar no es pobre y si tiene características físicas adecuadas, lo que se asocia con hogares de mayores recursos, y, al mismo tiempo, tiene menor probabilidad en los hijos más pequeños. 
Tabla 3.

Determinantes del sobrepeso infantil en El Oro

\begin{tabular}{|c|c|c|c|c|}
\hline $\begin{array}{l}\text { SOBREPESO } \\
\text { VARIABLE }\end{array}$ & $\begin{array}{l}\text { AÑOS } \\
2006\end{array}$ & & 2014 & \\
\hline \multirow[t]{2}{*}{ Rural } & 0,10 & $* *$ & $-0,02$ & \\
\hline & $(0,06)$ & & $(0,03)$ & \\
\hline \multirow[t]{2}{*}{ Pobre } & $-0,06$ & ** & $-0,06$ & ** \\
\hline & $(0,02)$ & & $(0,03)$ & \\
\hline \multirow[t]{2}{*}{ Agua red pública } & 0,01 & & 0,00 & \\
\hline & $(0,03)$ & & $(0,03)$ & \\
\hline \multirow[t]{2}{*}{ Servicio sanitario alc. } & 0,00 & & 0,02 & \\
\hline & $(0,03)$ & & $(0,03)$ & \\
\hline \multirow[t]{2}{*}{ Recolección basura mun. } & $-0,03$ & & 0,00 & \\
\hline & $(0,05)$ & & $(0,06)$ & \\
\hline \multirow[t]{2}{*}{ Techo adecuado } & 0,00 & & $-0,03$ & \\
\hline & $(0,02)$ & & $(0,02)$ & \\
\hline \multirow[t]{2}{*}{ Paredes adecuadas } & $-0,01$ & & 0,07 & * \\
\hline & $(0,04)$ & & $(0,03)$ & \\
\hline \multirow[t]{2}{*}{ Piso adecuado } & 0,03 & & $-0,03$ & \\
\hline & $(0,04)$ & & $(0,03)$ & \\
\hline \multirow[t]{2}{*}{ Vitamina A } & 0,02 & & 0,02 & \\
\hline & $(0,04)$ & & $(0,03)$ & \\
\hline \multirow[t]{2}{*}{ Hierro } & 0,01 & & $-0,03$ & \\
\hline & $(0,04)$ & & $(0,03)$ & \\
\hline \multirow[t]{2}{*}{ Edad } & 0,00 & & 0,00 & \\
\hline & $(0,00)$ & & $(0,00)$ & \\
\hline \multirow[t]{2}{*}{ Mujer } & $-0,04$ & $*$ & $-0,02$ & \\
\hline & $(0,02)$ & & $(0,02)$ & \\
\hline \multirow[t]{2}{*}{ Bajo peso } & & & $-0,04$ & \\
\hline & & & $(0,04)$ & \\
\hline \multirow[t]{2}{*}{ Orden nacimiento } & $-0,01$ & & $-0,03$ & $* *$ \\
\hline & $(0,01)$ & & $(0,01)$ & \\
\hline \multirow[t]{2}{*}{ Diarrea } & $-0,03$ & & $-0,03$ & \\
\hline & $(0,02)$ & & $(0,03)$ & \\
\hline
\end{tabular}




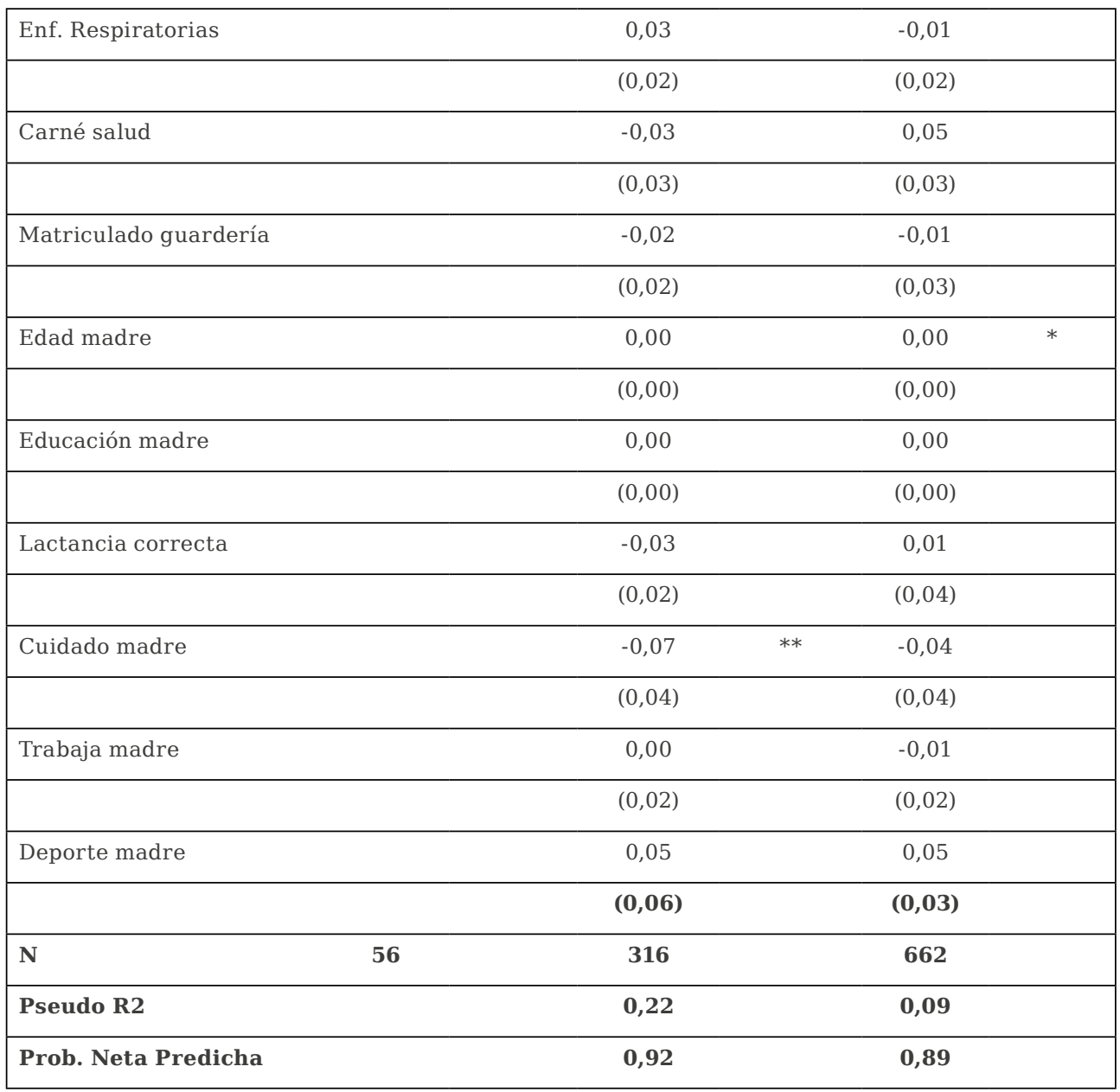

Con ello, los resultados indican que la provincia de El Oro presenta determinantes particulares de la malnutrición infantil, los cuales son distintos a los encontrados a nivel nacional y en otras provincias (Rivera, Olarte y Rivera, 2021; Rivera, Olarte y Rivera, 2020). En esa línea, es crucial resaltar la importancia del contexto para establecer estrategias alineadas con un combate a la malnutrición infantil.

Este estudio pretende aportar con insumos a la entrada en agenda pública de la importancia de la malnutrición infantil dentro de la provincia de El Oro, donde una de sus ventajas está en utilizar las cifras provinciales durante las últimas dos décadas, provenientes de fuentes oficiales, y abrir el camino para el desarrollo de políticas públicas específicas desde lo local para complementar lo nacional. En este proceso es fundamental la atención a los determinantes socioeconómicos de la malnutrición y generar un ambiente idóneo para un adecuado desarrollo infantil. 


\section{CONCLUSIONES}

La malnutrición infantil es un problema latente en Ecuador. Dentro de la provincia de El Oro, la malnutrición presenta niveles muy similares en desnutrición y sobrepeso, lo cual la convierte en la provincia con mayor paridad en este fenómeno, con niveles cercanos al 17\%, aunque con distintos determinantes.

\section{REFERENCIAS BIBLIOGRÁFICAS}

Berlinski, S., \& Schady, N. (2015). Los primeros años: el bienestar infantil y el papel de las políticas públicas. Washington: Banco Interamericano de Desarrollo.

Chávez, V., Fernández, N., Jaramillo, J., López, J., Mideros, A., Peña, C., Proaño, M., Romero, S., Uzcategui, M., \& Villacís, M. (2020). ¿Cómo está el desarrollo en Ecuador?, perspectivas desde el Plan de Desarrollo y Agenda 2030. Quito: Asamblea Nacional del Ecuador.

Freire, W. (2013). Resumen Ejecutivo. Tomo I: Encuesta Nacional de Salud y Nutrición. ENSANUT-ECU 2011-2013. Quito: Ministerio de Salud Pública/ Instituto Nacional de Estadística y Censos.

Freire, W. (2014). The double burden of undernutrition and excess body weight in Ecuador. The American Journal of Clinical Nutrition, 100(Suppl), 1634S-43S.

Instituto Nacional de Estadística y Censos. (2010). Fascículo provincial de El Oro. Quito: INEC.

Martínez, R., \& Fernández, A. (2009). El costo del hambre: impacto social y económico de la desnutrición infantil en el Estado Plurinacional de Bolivia, Ecuador, Paraguay y Perú. Santiago: CEPAL.

Organización Panamericana de la Salud. (2008). La desnutrición en lactantes y niños pequeños en América Latina y El Caribe: alcanzando los objetivos de desarrollo del milenio. Washington: OPS.

Prefectura de El Oro. (2015). Plan de Desarrollo y Ordenamiento Territorial. Machala: Prefectura de El Oro.

Rivera, J., Olarte, S., \& Rivera, N. (2021). La malnutrición infantil en Cotopaxi y sus determinantes. UTCiencia, 8(1), 148-155.

Rivera, J., Olarte, S., \& Rivera, N. (2021). Un problema crítico: la malnutrición infantil en Bolívar. Revista de Investigación Talentos, 8(1), 101-111.

Rivera, J., Olarte, S., \& Rivera, N. (2021). La malnutrición infantil en Tungurahua y sus determinantes. Revista Uniandes de Ciencias de la Salud, 4(1), 610-620.

Rivera, J., Olarte, S., \& Rivera, N. (2021). Un problema para el desarrollo: la malnutrición infantil en Los Ríos. Magazine de las ciencias: revista de investigación e innovación, 5(8), 90-99. 
Rivera, J., Olarte, S., \& Rivera, N. (2020). Madre hay una sola: malnutrición infantil en Carchi. Sathiri: Sembrador, 15(2), 291-300.

Rivera, J., Olarte, S., \& Rivera, N. (2020). Crónica de una muerte anunciada: la malnutrición infantil en Santa Elena. Ciencias Pedagógicas e Innovación, 8(1), 28-35.

Rivera, J., Olarte, S. \& Rivera, N. (2020). La Malnutrición Infantil en Esmeraldas: ¿Un éxito relativo?”. La U Investiga, 7(2), 68-78.

Rivera, J., Olarte, S. \& Rivera, N. (2020). El enemigo invisible: la malnutrición infantil en Imbabura. La U Investiga, 6(2), 1-8.

Rivera, J., Olarte, S. \& Rivera, N. (2020). Las secuelas del terremoto: el problema de la malnutrición infantil en Manabí. ECA Sinergia, 11(3), 40-49.

Rivera, J. (2019). La malnutrición infantil en Ecuador: una mirada desde las políticas públicas. Estudios de Políticas Públicas, 5(1), 7-30.

Senplades. (2014). Resultados de El Oro. Quito: Senplades.

Sundaram, J. (2015). Ending Malnutrition: from commitment to action. FAO: Roma.

WHO (2006). Child Growth Standards. WHO: Geneva. 